\title{
Research on the Communication Effect of Emoji Derived from Hot Topics on Social Media
}

\author{
Yuting $\mathrm{Li}^{1{ }^{1 *}}$ Meijie $\mathrm{Wu}^{2}$ \\ ${ }^{1}$ School of Art and Design, Guangdong University of Finance and Economics, Guangzhou, Guangdong, China \\ ${ }^{2}$ School of Advertising, Communication University of China, Beijing, China \\ *Corresponding author. Email: Yuting_Li@gdufe.edu.cn
}

\begin{abstract}
Emojis in the social media era have become a vital part of people's online social activities, and their development has continuously enhanced people's demand for online communication experience. This article sorts out the hot topics that appear on Weibo in 2020, selects the hot topics in the two major areas of business and daily life, and analyzes the use of Emojis and the communication effects achieved under hot topics in different fields. This paper first uses Octopus data collector to collect blog posts and blog comments under the three major topics, then uses ROST CM 6.0 to perform word frequency statistics and semantics analysis on the collected data, and discusses the communication effect of Emoji of hot topics through qualitative methods. Companies in the commercial sector use Emojis to deepen the personification image of brands. In people's daily lives, Emojis provide a window for netizens to express themselves and seek empathy.
\end{abstract}

Keywords: Internet hot topics, Emoji, Text analysis method, Communication effect.

\section{INTRODUCTION}

Under the impact of the Internet, social media has risen rapidly, and people's lives and communication methods are undergoing earthshaking changes. Taking WeChat and QQ, the instant messaging tools most used by Chinese netizens, as the examples, the way of using them has changed from the initial text and voice communication to the current "emoji battle". In the era in which "everyone has a microphone", netizens are constantly discussing hot topics on the Internet.[1] Compared with the previous text discussion and dissemination, Emojis have gradually become a new way of expressing hot topics and have been widely disseminated in cyberspace, achieving remarkable communication effects and becoming an important communication tool indispensable for subjects in different fields to achieve their own goals and motivations.

*Fund: This article is supported by the results of 2020 Guangdong Provincial Degree and Graduate Education Reform Project - "Research on the Management Mechanism of the Joint Training Demonstration Base Construction of Graduate Education Based on Process Control" (2020JGXM052).

\section{RESEARCH METHODS AND DATA SOURCES}

In the selection of hot topics on the Internet, the criteria are as follows: first, the case topics arouse hot discussion and attention from netizens, and netizens have interactive forms such as transmitting and commenting; second, the selected hot topics derive relevant Emojis, which are widely used and discussed in social platform and the social activities of netizens; third, case topics cover different fields. [2] This article selects 2 cases that meet the criteria from a large number of hot topics to make the classification and summary. They are Alibaba's educational software "Online Begging of DingTalk for Mercy" and the "laborer" to express themselves. By collecting online comments on these topics on Weibo from netizens, it is required to analyze the communication effects of Emojis of hot topics in different fields.

In this paper, the network data collection software "Octopus data collector" is used for data collection, and the ROST CM 6.0 is used to perform word frequency statistics and semantic analysis on the collected data.[4] Based on this, the 
communication effect of hot topic Emojis is analyzed.

\section{ANALYSIS OF THE COMMUNICATION EFFECT OF EMOJIS DERIVED FROM HOT TOPICS IN THE TWO FIELDS}

The communication effect has dual meanings. In the narrow sense, the communication effect refers to degree that the communication activity has achieved the communicator's intention or purpose. In the broad sense, the communication effect refers to the overall impact and results of the communication activity, especially the newspapers, radio, and television on the recipient and the society.[4] The communication effect of hot topic emoji studied in this paper refers to the effect in a narrow sense. Communication effect can also be divided into different levels. According to the consensus of scholars, communication effect can be at cognitive level, attitude level and behavior level. Through the analysis of online comments and related blog posts of netizens, and taking the cognition, attitude and behavior of netizens as the

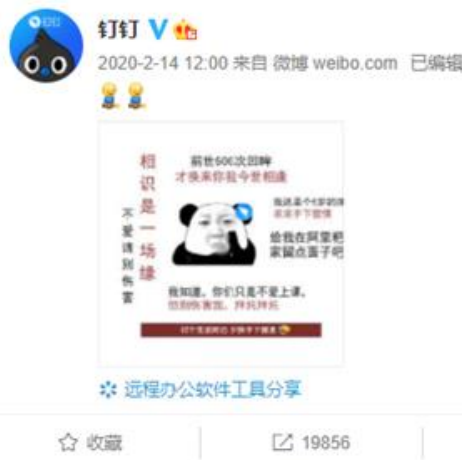

Figure 1 Dynamic screenshot of the official blog.

The author captured 500 online comments of this blog post for word frequency analysis and semantic analysis to study its communication effect.("Figure 1")

\subsubsection{Word Frequency Analysis and Semantic Analysis}

\subsubsection{Word Frequency Analysis}

"Five-star praise by installments", the highfrequency word on the cognitive level of netizens, is a hot "topic" on the Internet. The self-made emojis also show the request of "young man giving the stars generously". From the word frequency measurement indexes, this paper studies the communication effects of Emojis derived from hot topics in different fields.

\subsection{Business Field: Educational Software Carries out Crisis Public Relations with the Help of Emojis}

\subsubsection{Overview of the Topic of Online Plea for Mercy and Derivative Emojis}

DingTalk is a software owned by Alibaba Group. During the COVID-19 epidemic in 2020, the Ministry of Education of China selected DingTalk as a platform for students to attend classes. During the online class, a large number of students were required to clock in several times a day, the amount of homework increased compared with that in the period of offline teaching. However, the students could not venting to the school and the teacher, the spearhead was aimed at the online class APP. In the download center, they gave one-star evaluation of "five star praise by installments". Therefore, DingTalk became "the worst" software.

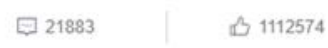

table, it can be seen that "five-star praise, one star, installment" appears frequently."Application, high prasie, having classes" and other words also appear in high frequency, indicating that netizens are relatively familiar with the functional characteristics of DingTalk.

On the attitude level of netizens, high frequency words, such as "child, fighting, poor, love early, hug", appear more often, showing that the netizens tend to respond positively to the sadfishing. The netizens say that DingTalk is just a "child".

On the behavior level of netizens, high frequency words include "speciall, high praise, pay off, the first time". The related comments are as 
follows: "I paid off in a single payment, I do not give five stars by installments, and please quick praise me"; "this is the first time to comment on the software, and I give 5 stars." Netizens "love" DingTalk, and download the software to give a high score for the evaluation. ("Table 1")

Table 1. Word frequency diagram of comments on official blog posts

\begin{tabular}{ccc|ccc}
\hline serial number & comment & frequency & serial number & comment & frequency \\
\hline 1 & five-star praise & 214 & 14 & lovely & 30 \\
2 & applicantion & 104 & 15 & poor & 29 \\
3 & teacher & 77 & 16 & specially & 20 \\
4 & high praise & 77 & 17 & pupil & 18 \\
5 & having classes & 59 & 18 & hug & 15 \\
6 & one star & 56 & 19 & Love dearly & 15 \\
7 & living lesson & 48 & 20 & intallment & 14 \\
8 & student & 42 & 21 & pay off & 13 \\
9 & homework & 38 & 22 & gratitude & 12 \\
10 & fighting & 37 & 23 & appraise & 12 \\
11 & child & 37 & 24 & arrange & 8 \\
12 & school & 36 & 25 & the first time & 8 \\
13 & function & 31 & &
\end{tabular}

\subsubsection{Semantic Graph Analysis}

On the whole, Semantic Web Graph presents a "core-edge" structure, which can be divided into four levels. The first level is the core level, and there are words such as "five-star praise, application, Ding Talk", which are closely related to the "stalk" in the topic of "online begging for forgiveness". The second level is the sub-core level, and there are words such as "student, school," etc., which are mainly related to the subjects involved in the topic of "online plea for mercy". The third level is the marginal level, and there are words such as "poor, child, specially, function" etc., mainly referring to netizens' comments on the topic and the incident itself. "Convinence" appeared in the marginal level of the semantic graph, specifically being regarded as "application - having classes convinence", which reflected that netizens tended to have positive evaluation on the function of DingTalk.In the marginal level, the words "poor" and "specially" are as follows: "poor - five-star praise", and "specially - five-star praise". And the specific comment is that "Don't worry, DingTalk. I download to give five-star praise specially." ("Figure 2")

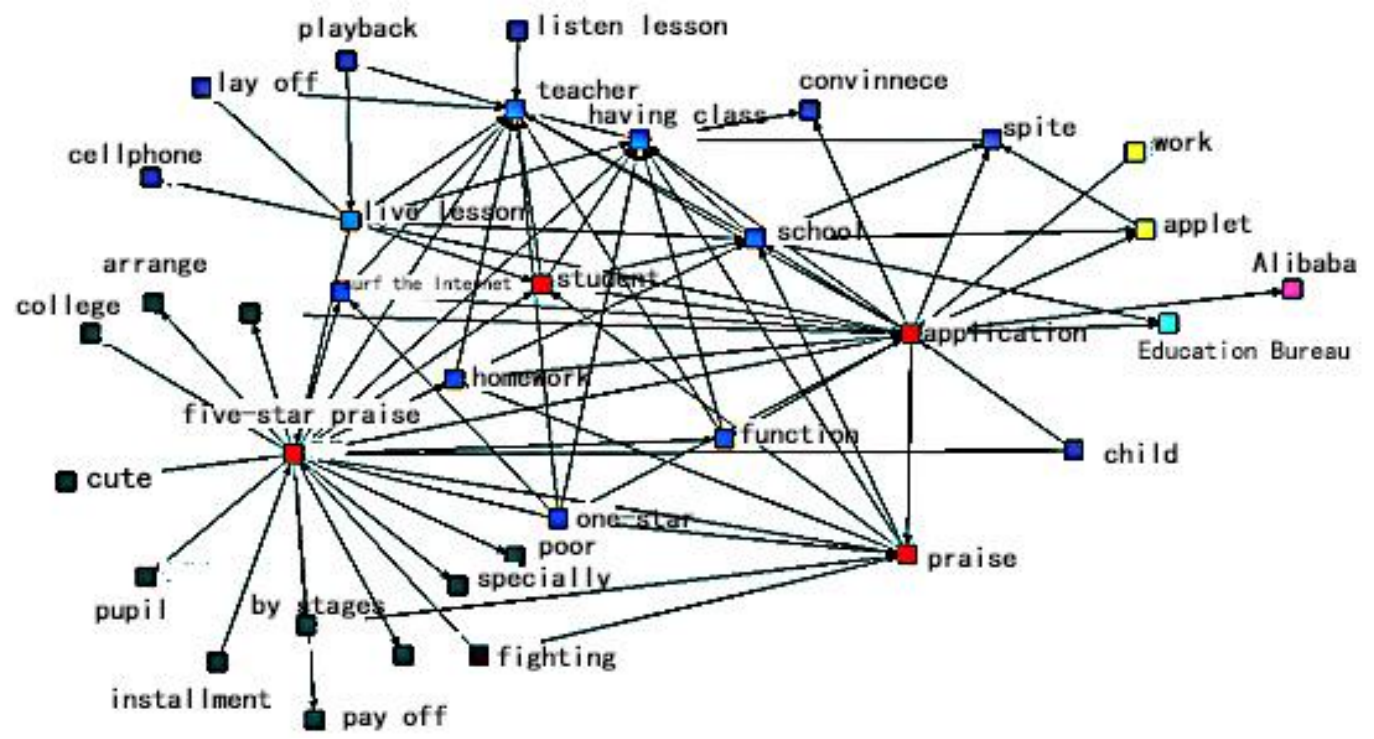

Figure 2 Semantic graph of comments on official blogs. 


\subsubsection{Analysis of Communication Effect: Personalization of the Brand Narrates the Distance with Consumers}

Using emojis and the heat of the event, DingTalk built on past personifications to portray itself as a wronged "child image", squatting down to talk to its audience rather than adopting a serious official response.

According to the word frequency graph, the high frequency words that appear at the attitude level of the netizens include 'child', 'fighting', 'poor', 'love early', 'hug'. The image of the 'aggrieved child' that DingTalk conveys through the emojis has received positive responses from the netizens, which has strengthened the personified image of DingTalk. DingTalk is no longer a "ruthless" teaching tool, but has become a "poor, weak and helpless swallow in the public mind, and it just wants to obtain "five-star" praise. In the semantic graph, about "poor — five-star praise" and "specially - five-star praise", the specific comments are as follows: "Don't cry, I specially download to give five-star praise"; "I will go to make five-star praise"; and "the world's most poor software is Dingtalk". These comments once again verify the deep popularity of the personification image of Dingtalk. When the public see the aggrieved Dingtalk, netizens began to take action — giving five-star praise.

In the crisis public relations, Dingtalk creates the image of a failure through the expression of "sadfishing", which is widely spread on social media. The attitudes of netizens for Dingtalk have changed in the aspects of cognition, attitude and behavior. The personification image of Dingtalk is popular among people, breaking the asymmetry between the enterprises and ordinary users, narrowing the distance between enterprisess and consumer, andimproving the transmission and reputation of Dingtalk.

\subsection{Daily Life: the Employees Express Themselves and Seek the Resonance Through Emojis}

\subsubsection{Overview of the Topic of "Employee" and Its Derivative Emojis}

In October 2020, the hashtag \# Worker \# flooded every major social media platform in China. In a short video posted by web celebrity blogger "Abstraction and Basket", there are many similar messages, for example, "hardworking people are already running up the tower crane, but you stretch yourself in the quilt. You don't take your life seriously. Good morning, employee!" These "online messages" are serious and full of ridicule, causing a large number of netizens to forward and imitate the creation on social platforms.[5] By February 2021, the hashtag \# Employee Emoji \# has been read 170 million times on Weibo. ("Figure 3")

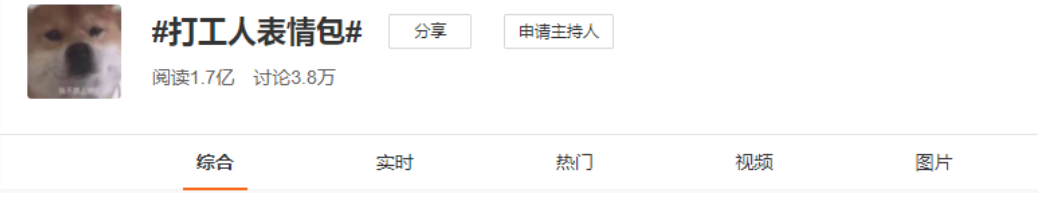

Figure 3 Details of \# employee emojis \#.

\subsubsection{Word Frequency Analysis and Semantic Graph Analysis}

resonance" also have relatively high word frequency. ("Table 2")

\subsubsection{Word Frequency Analysis}

According to the word frequency table, the words with the highest word frequency are the core words of the topic, such as "commuter, good morning, employee, boss", etc., and the words with the second highest word frequency are the more inspirational words, such as "effort, fighting, struggle". Also, words of "self-mockery and 
Table 2. Word frequency diagram of comments on related blog posts about the topic of \# employee \#

\begin{tabular}{ccc|ccc}
\hline serial number & comment & frequency & serial number & comment & frequency \\
\hline 1 & commuter & 1208 & 14 & income & 17 \\
2 & reason & 116 & 15 & rent & 16 \\
3 & recently & 114 & 16 & reality & 15 \\
4 & good moring & 61 & 17 & self-mockery & 14 \\
5 & employee & 58 & 18 & plan & 13 \\
6 & boss & 30 & 19 & popular & 13 \\
7 & effort & 25 & 20 & joy & 13 \\
8 & fighting & 21 & 21 & work overtime & 13 \\
9 & life & 19 & 22 & go on duty & 13 \\
10 & youngster & 19 & 23 & waste & 12 \\
11 & struggle & 18 & 24 & time & 12 \\
12 & Shanghai & 18 & 25 & resonance & 12 \\
13 & salary & 17 & & & \\
\hline
\end{tabular}

\subsubsection{Semantic Graph Analysis}

It can be seen from the semantic map that the words at the core level, such as "commuter, effort, fighting, struggle", have a semantic connection with "commuter", and the word "self-mockery" appears in the marginal level of the semantic map, which is more detailed as " "self-mockery-commuterhelpness". It reflects that netizens express helplessness through self-deprecating, and "renson-find out-resonance" reflects that netizens can find resonance through the use of employee stalk and employee emojis. ("Figure 4")

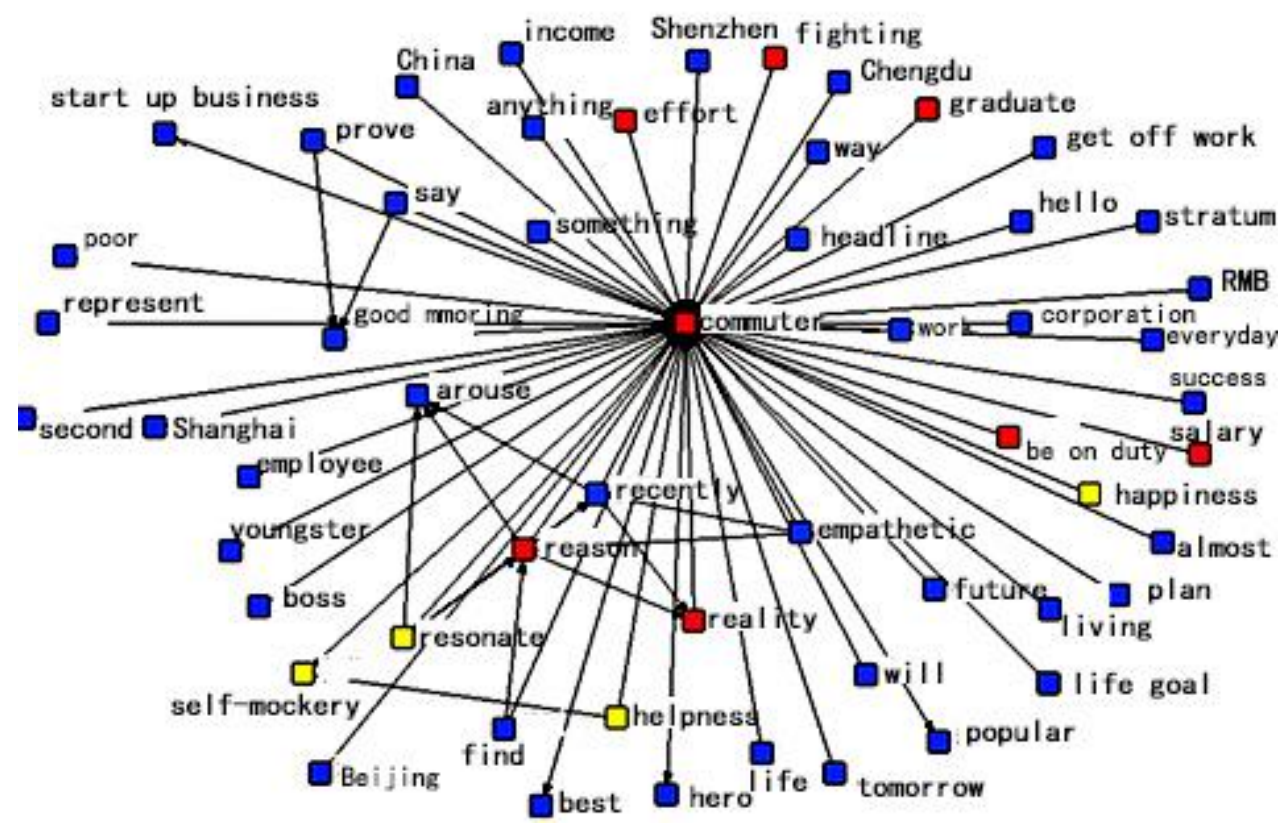

Figure 4 Semantic graph of comments on related blog posts about \# employee topic \#.

\subsubsection{Analysis of Communication Effect:}

Grassroots People Express Themselves and Seek Resonance

In the word frequency graph, words such as "commuter, employee, effort, and struggle" appear more frequently, reflecting that netizens tend to add motivational words to motivate themselves when they call themselves employees, forming positive- energy employees' quotations, and taking emojis as the carrier of communication. At the same time, there are more frequent occurrences of "selfmockery, resonance", etc. In the semantic map, "self-mockery-commuter-helpness" all reflect that behind the inspiring sayings of the workers, people actually want to express the helplessness and hardships of work and life. They hope to express themselves and express their emotions by 
means of self-mockery and carnival; "reasonfind-resonance-empathetic" can reflect the workers of different industries and different incomes claim to be "employees". They are seeking an identity and a sense of belonging.[5] The employeess who use the workers' quotations express their status in a joking way. In a highpressure society, both the "elites" and the "corporate slaves" need to be sad for their livelihoods. At the same time, netizens also build their own subcultural circle by means of "working workers" and making a large number of emojis. In the process of consumption and production, it is required to strengthen the identity and sense of belonging to the circle to which they belong, and find emotional resonance.

\section{CONCLUSION}

As a new type of discourse expression tool, emojis have more and more profound effects on people's social activities and online communication. As a member of the emoji army, emojis derived from online hot topics have also received more and more attention. This paper selects two representative topics in the network to analyze the communication phenomenon and effect of emojis. It is found that the application fields of emojis derived from the hot topics in the network are more and more extensive, involving business and daily life. Although the change speed of the emojis of hot topics is fast, its communication range is quite wide during the duration of hot topics, and it plays an important role in boosting hot topics. At the same time, it has achieved remarkable results in helping enterprises to shape the brand personality image, breaking the stereotype of the official mainstream media, and helping grassroots people to express themselves out of the mainstream bondage. However, it is worth noting that there are also some problems in the use of hot topic emojis, such as the proliferation of vulgar emojis and serious infringement. This requires the joint efforts of many parties. Relevant departments have issued corresponding explicit provisions to stop it.[6] The platform has strengthened the shutdown system to prevent the transmission of bad emojis. Internet users should improve their media literacy to identify the types of emojis. Only through the efforts of all parties can the new culture develop in the right way.

\section{AUTHORS' CONTRIBUTIONS}

Yuting $\mathrm{Li}$ contributed to topic design and revising and editing, Meijie $\mathrm{Wu}$ is responsible for research design and wrote the manuscript.

\section{REFERENCES}

[1] Zhang Ning. Dispelling as resistance: An analysis of the youth subculture of the "Internet meme war" [J]. Modern Communication (Journal of Communication University of China), 2016. (in Chinese)

[2] Li Fangze. Generation and application of hot topic expression package in network public space. [D]. Shenyang Normal University, 2020 (in Chinese)

[3] Sun Xiaodong, Ni Rongxin. Chinese cruisers' product cognition, emotional expression and brand image perception [J]. Geographical Research, 2018. (in Chinese)

[4] Guo Qingguang. Communication course: Second Edition [M]. Beijing: China People's publishing house, 2011. (in Chinese)

[5] Wei Xiaoyang. Incorporation and resistance: a study of "Employee"topic from theperspective of youth subculture communication Interpretation [J]. Audio visual, 2021. (in Chinese)

[6] Zhuang Shanying, Zou Hui. Communication and reflection of expression pack in the new media era [J]. Audio visual, 2020. (in Chinese) 